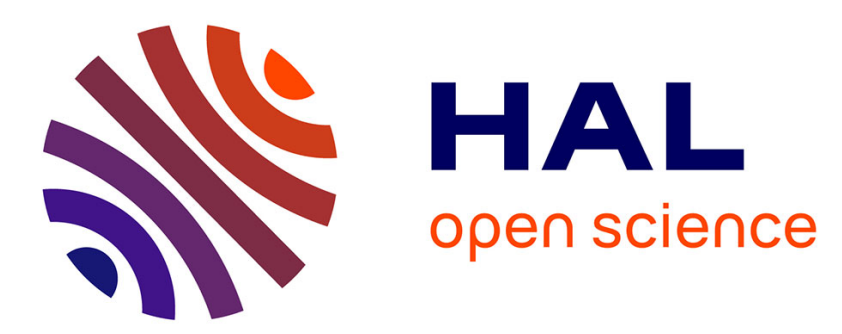

\title{
Incentive scheduler algorithm for cooperation and coverage extension in wireless Networks
}

Cédric Gueguen, Abderrezak Rachedi, Mohsen Guizani

\section{To cite this version:}

Cédric Gueguen, Abderrezak Rachedi, Mohsen Guizani. Incentive scheduler algorithm for cooperation and coverage extension in wireless Networks. IEEE Transactions on Vehicular Technology, 2013, 62 (2), pp.797-808. 10.1109/TVT.2012.2225855 . hal-00740058

\section{HAL Id: hal-00740058 \\ https://hal.science/hal-00740058}

Submitted on 16 Oct 2012

HAL is a multi-disciplinary open access archive for the deposit and dissemination of scientific research documents, whether they are published or not. The documents may come from teaching and research institutions in France or abroad, or from public or private research centers.
L'archive ouverte pluridisciplinaire HAL, est destinée au dépôt et à la diffusion de documents scientifiques de niveau recherche, publiés ou non, émanant des établissements d'enseignement et de recherche français ou étrangers, des laboratoires publics ou privés. 


\title{
Incentive Scheduler Algorithm for Cooperation and Coverage Extension in Wireless Networks
}

\author{
Cédric Gueguen, Member, IEEE, Abderrezak Rachedi, Member, IEEE, and Mohsen Guizani, Fellow, IEEE
}

\begin{abstract}
In this paper, we focus on the wireless coverage extension and nodes' cooperation. We propose a new protocol based on an incentive approach and a scheduling algorithm in order to reward cooperative nodes. The cost of cooperation can be prohibitively expensive in terms of QoS and energy consumption which does not motivate some nodes to cooperate. Therefore, we introduce a percentage of cooperation and QoS parameters in the scheduling algorithm called $C E I$ in order to incite potential mobile relaying nodes to cooperate and in turn extend the wireless areas. We use the cross-layer approach in order to optimize the QoS parameters. The proposed solution does not only incite the nodes to cooperate but also enhance the QoS by increasing the average throughput and decreasing the delay. The simulation results show that the proposed solution does not only give better results than the well known scheduling algorithms like MaxSNR and WFO but also allows the cooperative mobile nodes to increase their own throughput by around $114 \%$. The total amount of data transmitted out of the cell in order to extend the coverage can be increased by around $59 \%$ compared to the scheduling algorithm MaxSNR.
\end{abstract}

Index Terms-Coverage extension, Incentive Scheduling, Cooperation, Selfish nodes, Quality of Service, Multipath fading.

\section{INTRODUCTION}

The basic purpose of the coverage extension area in wireless networks is to increase the network connectivity without increasing the infrastructure. This is one of the main applications of cooperative communications in wireless networks. The coverage extension issue requires the cooperation of border mobile nodes to relay the packets of neighbouring nodes that are located outside the base-station area. For instance, the nodes located at two hops from the Access Point (AP) can access the services offered by the AP through the relayed nodes like Internet as illustrated in figure 1. Many researchers worked on strategies to find the optimal placement for the relayed nodes in order to guarantee a high Quality of Services (QoS) [1]. Other works dealt with the optimal number of hops between relayed nodes in wireless networks [2][3]. However, they assume that the relayed nodes by definition are fixed and cooperative, which is not interesting in the case of a dynamic wireless network where the nodes freely move and may be selfish.

Copyright (c) 2012 IEEE. Personal use of this material is permitted. However, permission to use this material for any other purposes must be obtained from the IEEE by sending a request to pubs-permissions@ieee.org.

C. Gueguen is associate professor with the IRISA Lab.(UMR 6074), University of Rennes 1, Rennes, France. E-mail: cedric.gueguen@irisa.fr

A. Rachedi is associate professor with the Gaspard Monge Computer Science Laboratory (LIGM UMR8049), University Paris-Est, Champs-surmarne, France. E-mail: rachedi@ieee.org

M. Guizani is full professor with Qatar University, Qatar. E-mail: mguizani@ieee.org

Manuscript received June 06, 2012; revised September 16, 2012; accepted October 01, 2012.
The mobility of relayed nodes has to be taken into account in order to be close to reality. Other works use mobile relayed nodes to extend the wireless coverage with throughput enhancement [4][5]. However, no incentive approach is considered in the latter works. The relayed nodes must share their throughput with other neighbouring nodes that can impact their own packets' transmission. In addition, the energy consumption of the relayed nodes is more important than the one of other classical nodes. They do not only transmit their own packets but also the packets of other neighbouring nodes. Therefore, the user of potential relayed nodes can disable the cooperative functionality in order to keep the performance in terms of QoS only for its own transmission. In this paper, we consider that mobile relayed nodes are not part of the fixed wireless infrastructure. That is why the incentive strategy for potential mobile relay nodes has to be taken into account in the cooperation protocol design. The main incentive models discussed in the literature are based on game theory [6][7][8][9]. However, it is hard to implement these models because of some assumptions and because no implementation or performance evaluation is given. We believe that the scheduling algorithms can tackle this problem by adapting and introducing new parameters like incentives with QoS. Moreover, the scheduling algorithms are already implemented in the Access Point and in routers thus facilitating our study.

\section{A. Contributions}

In this paper, we propose a new cooperative protocol based on an incentive approach that takes into account the QoS for mobile relayed nodes in order to extend the coverage area. This approach consists of increasing the priorities of the relayed nodes according to their cooperation rate. The idea is to reward the relayed nodes for their cooperation instead of penalizing them by increasing the cost of cooperation. Consequently, the nodes have no interest in selecting and acting selfishly, by using their throughput only to transmit their own packets. Moreover, our protocol guarantees that the nodes are free to cooperate, because they choose their percentage of cooperation. The proposed solution combines the QoS parameters and cooperation rate using the crosslayer approach with a scheduling algorithm. This solution is called Coverage Extension based on Incentive scheduling $(C E I)$. Moreover, the physical layer information is used in order to take advantage of the time, frequency and multiuser diversity and to optimize the system capacity until it is close to the Shannon limit. Unlike some existing models, our solution can be widely implemented. In addition, we present the performance evaluation of our solution in terms of delay, throughput and relaying efficiency with different cooperation ratios of nodes. The comparison between the proposed CEI and other 


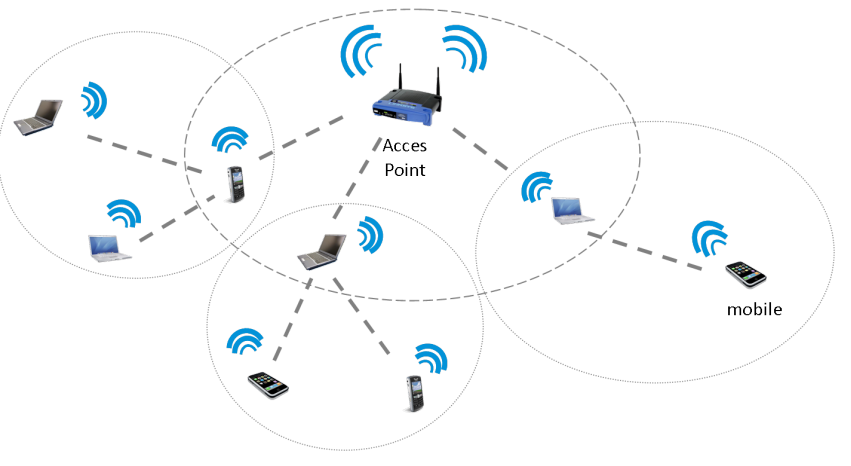

Fig. 1. Coverage extension in wireless network.

existing resource allocation strategies like the classical RoundRobin (RR) [10], acknowledged MaxSNR [11][12] and WFO [13] are presented and analyzed.

\section{B. Organization}

This paper is organized as follows: in Section 2, we present the existing works related to coverage extension using cooperation in wireless networks, incentive models and scheduling algorithms. Section 3 provides a detailed description of the system under study and describes the proposed coverage extension protocol based on the incentive scheduler. The fourth section presents the obtained simulation results and their analysis. Finally, Section 5 concludes the paper and presents our future works.

\section{RELATED WORK}

In this section, we present existing works related to coverage extension protocols, cooperation incentives models and scheduling algorithms.

\section{A. Coverage extension Protocols}

Wireless coverage extension is one application of the cooperation communications system. Many existing works deal with the coverage extension by analysing the different strategies to find the optimal placement for the relayed nodes in order to guarantee a high Quality of Services (QoS). Sadek et al. [1] proposed two distributed relay-assignment protocols in order to reduce the outage and increase the network connectivity. The first protocol selects the relayed node that is best placed while taking into account the quality of SNR and the distance between nodes. The second protocol gives the optimal placement for the fixed relayed nodes so that they help the existing users. Other works deal with the optimal number of hops between relayed nodes in multi-hop wireless networks. Florea and Yanikomeroglu [2] have shown that the optimal number of relayed nodes can be determined for multihop link under the assumption that all links have the same path loss exponent and that the relays are located at equal intervals. Only a few works propose to use the mobile relayed nodes to extend the wireless coverage and enhance the throughput. Xiao et al. [4] propose quantitative studies of benefits offered by mobile relayed nodes for a potential coverage area extension. The mobile node relays offer substantial coverage extension benefits. However, no incentive approach is considered in these works and they assume that the relayed nodes are all cooperative.

\section{B. Cooperation Incentives Models}

Two types of uncooperative nodes can be distinguished: the malicious nodes and the selfish nodes. The malicious nodes try to attack the system by choosing an uncooperative behaviour and creating a network disconnection. The goal of the selfish nodes is to maximize their benefits in terms of QoS (like throughput and delay) and to minimize their costs like the energy consumption. In this paper, we focus on the selfish behaviour of potential cooperative nodes. The cooperation is an important parameter in wireless networks, because without any packet forwarding the ad hoc network cannot exist and the wireless coverage extension is not possible.

The concept of cooperative communication (CC) technique in wireless networks was introduced in [14]. In literature, two main solutions were proposed to overcome the problem of selfish nodes. The first one is based on the reputation mechanisms that consist in assessing a nodes' contribution to the network, like its forwarding and routing functionalities [15][16][17][18][19]. The reputation model called CONFIDANT was proposed to share the reputation metric and alarm messages in order to detect and punish the misbehaving nodes [17]. Another model called CORE is proposed to implement the reputation function by using the monitoring technique. Each node computes the reputation value of its neighbour and refuses to provide any service to misbehaving nodes when their reputation is lower than a certain threshold [15]. However, these solutions neither overcame the problems of false observation related to collisions nor considered the performance of potential relayed nodes. In [19][20] the authors introduce the concept of cross-layer in order to reduce the false observation rate related to collisions, but no incentive model is proposed.

The second one is based on economic mechanisms like price-based and game theories [8][9] [21][22]. In these models nodes are paid to offer message forwarding services and also pay to receive forwarding services. These proposed incentive models based on the price and game theories have introduced the concept of virtual cash. The nodes are rewarded for packets forwarding by trading virtual cash with source and next hop nodes. Buttyan and Hubeaux [23] proposed nuglets as credits to manage forwarding transactions. The source node pays relay intermediate nodes by storing a nuglet in the packet head. The intermediate nodes acquire the nuglets when forwarding the packets. In [24] a hybrid model used the reputation metric and the price-based mechanism was proposed to overcome the issue of selfish nodes. However, the implementation of these solutions in resource allocation schedulers is not easy and the model assumptions must be adapted. That is why we propose a new scheduling algorithm based on QoS and the incentive parameters in order to reward cooperative mobile nodes. The scheduling algorithms are already implemented in Access Point and in routers. Their implementation can be carried out with a performance evaluation. 


\section{Scheduling Algorithms in Wireless Networks}

1) Maximum Signal-to-Noise Ratio Scheduling: The conventional access methods like Round Robin (RR) [10] and Random Access (RA) [25] are not adapted to the wireless environment and provide poor throughput. More recently intensive research efforts have been made in order to propose more efficient schedulers: opportunistic schedulers. They preferably allocate resources to active mobile(s) with the most favourable channel conditions at a given time. One major scheduling algorithm has emerged and appeared in the literature as a reference: the Maximum Signal-to-Noise Ratio (MaxSNR)[11][12].

Denoting $m_{k, n}$ the maximum number of bits that can be transmitted on a time slot of Resource Unit (RU) $n$ if this $\mathrm{RU}$ is allocated to mobile $k$, MaxSNR scheduling consists in allocating RU $n$ to mobile $j$ which has the greatest $m_{k, n}$ such as:

$$
j=\operatorname{argmax}_{k}\left(m_{k, n}\right), k=1, \ldots, K,
$$

with $K$ as the number of mobiles in the access point coverage zone.

Benefiting from multiuser and frequency diversity, MaxSNR scheduling continuously allocates radio resources to the mobile that has the best spectral efficiency. Consequently, MaxSNR strongly increases the system throughput. Dynamically adapting the modulation and coding that allows one to always make the most efficient use of the radio resources and to come closer to the Shannon limit. However, MaxSNR does not take into account any other aspect than the throughput. Indeed, MaxSNR scheduling does not manage priorities in order to favour cooperative mobiles. Consequently, the cooperative mobiles have no guaranteed reward. Their supplementary energy consumption and the personal throughput loss are not compensated. These results show that cooperation means penalty, and thus they do not encourage any cooperative network or coverage extension.

2) Weighted Fair Opportunistic Scheduling: We have recently proposed a new MAC scheduler called Weighted Fair Opportunistic (WFO) for an efficient support of multimedia services in multi-user OFDM wireless networks [13], [26]. Built in accordance with a cross-layer approach, this scheme is designed to benefit from the multi-user diversity while taking advantage of the dynamics of the multiplexed traffic. It takes into account both the transmission conditions in order to maximize global cell throughput and the higher layer constraints (such as traffic patterns, QoS constraints) in order to to ensure the same QoS level to all mobiles whatever the context.WFO dynamically favours the mobiles that go through a critical period in terms of QoS requirements, by using dynamic priorities.

The meaningful constraint regarding delay is the limitation of large values occurrences. In [13], we define the concept of delay outage by analogy with the concept of outage used in system coverage planning. A mobile $k$ is considered in delay outage (in a critical period) when its packets experience a delay greater than a given threshold defined by the mobile application requirements. The Packet Delay Outage Ratio
(PDOR) of mobile $k\left(P D O R_{k}\right)$ represents the emergency for mobile $k$ to be served. A mobile can be considered satisfied when, at the end of its connection, its delay constraint is met, i.e. its $P D O R$ experienced is less than a PDOR target specific to the mobile application.

The WFO scheduling principle is to allocate a Resource Unit $n$ to mobile $j$ which has the greatest WFO parameter value $W F O_{k, n}$ with:

$$
j=\operatorname{argmax}_{k}\left(W F O_{k, n}\right), k=1, \ldots, K,
$$

where $W F O_{k, n}$ is equal to:

$$
W F O_{k, n}=m_{k, n} \times f\left(P D O R_{k}\right),
$$

with $f$ a strictly increasing polynomial function defined in [13].

With this original weighted system, WFO keeps a maximum number of flows active across time but with relatively low traffic backlogs which results in a well-balanced resource allocation. Preserving the multiuser diversity allows to continuously benefit from opportunistic scheduling and thus maximize the bandwidth usage efficiency. The results have shown that WFO better conceals the system capacity maximization, QoS support and fairness objectives than MaxSNR scheme. WFO tackles the fairness problem between mobiles that have different cooperation ratios. However, we can notice that even if the cooperative nodes benefit from the same quality as selfish nodes, they are not rewarded for their supplementary energy consumption.

In this paper, unlike the existing scheduling algorithms like MaxSNR and WFO, we introduce the an incentive approach in order to reward the cooperative nodes and to balance their energy consumption by increasing their priority in terms of ressource allocation.

\section{Coverage Extension Protocol}

\section{A. Preliminaries}

In this subsection, we give some definitions and the wireless network context. We focus on the coverage extension of the Wireless Local Area (WLAN) and particularly of the access point area using the allocation of radio resources while considering a cooperative behaviour. However, the proposed solution can be applied to the Mobile Ad hoc Networks (MANETs) context under one condition, that is to use the cluster-based architecture. Figure 2 illustrates an example of the radio resources allocation among nodes located in the Access point coverage area.

We consider a centralized approach based on access point in WLAN or on cluster-head in MANETs. Indeed, maximizing the system capacity is one of the most crucial issues of wireless networks and a centralized approach is needed to allow an opportunistic scheduling which provides significant system throughput gains compared to a decentralized resources allocation. The packets originating from the backhaul network are buffered in the AP which schedules the downlink transmissions. In the uplink, the mobiles signal their traffic backlog to the access point which builds the uplink resource mapping. 


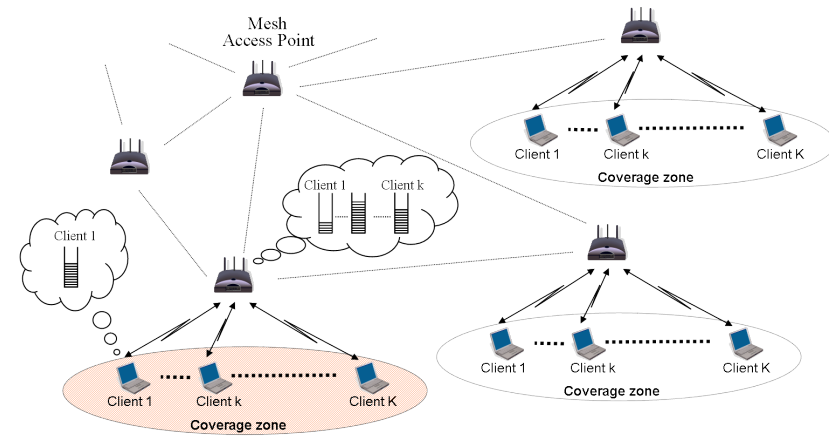

Fig. 2. Allocation of radio resources among the set of mobiles situated in the coverage zone of an access point.

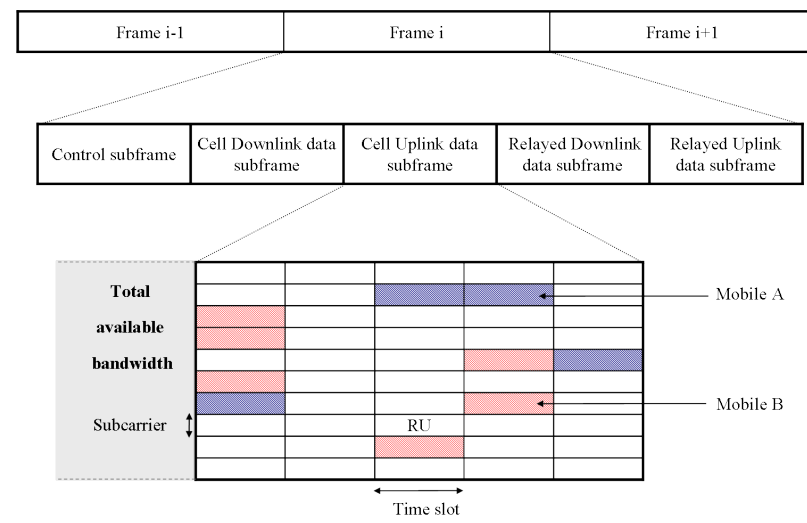

Fig. 3. Frame structure in TDD mode.

We assume that the physical layer operates using the structure described in Fig. 3. The total available bandwidth is divided into sub-frequency bands or subcarriers. The radio resource is further divided into frames in the time domain. Each frame is itself divided into time slots of constant duration. The time slot duration is an integer multiple of the OFDM symbol duration. Moreover, the frame duration is fixed to a value much smaller than the coherence time (inverse of the Doppler spread) of the channel. With such assumptions, the transmission on each subcarrier is subject to flat fading with a channel state that can be considered static during each frame.

The elementary Resource Unit is defined as any (subcarrier, time slot) pair. Each of these RUs may be allocated to any mobile with a specific modulation order. Transmissions performed on different RUs by different mobiles have independent channel state variations [27]. On each RU, the modulation scheme is QAM with a modulation order adapted to the channel state between the access point and the mobile to which it is allocated. This provides the flexible resource allocation framework required for an opportunistic scheduling.

The system operates using time division duplexing with five subframes: the control subframe, the cell downlink data subframe, the cell uplink data subframe, the relayed downlink data subframe and the relayed uplink data subframe. The cell uplink and downlink data subframes are used for the transmission of intra-cellular user data while the relayed uplink and downlink data subframes are used for the transmission by the relaying nodes of extra-cellular user data. During the control subframe, the access point sends control information towards its mobiles. This control information represents the scheduler decision which is constituted of three main parts: the resources mapping, the selected modulation order, and the selected emission power. In addition, during this subframe, the active mobiles send their current traffic backlog and information elements such as transmission power. This subframe is also used by the mobiles to establish their connections. This frame structure supposes a perfect time and frequency synchronization between the mobiles and the access point as described in [28]. Therefore, each frame starts with a long preamble used for synchronisation purposes. Additional preambles may also be used in the frame.

\section{B. The Incentive Scheduler Algorithm}

The main element of the proposed protocol is its scheduling algorithm called CEI. The scheduler, located in the central node like access point or cluster-head node, grants RUs to each mobile as a function of: (1) its channel state, (2) its current cooperation ratio, (3) its network confidence percentage, (4) its traffic backlog.

The channel state is supposed to be available at the receiver [29]. The current channel attenuation on each subcarrier and for each mobile node is estimated by the access node based on the SNR of the signal sent by each mobile during the uplink contention subframe. Assuming that the channel state is stable on a scale of $50 \mathrm{~ms}$ [30], and using a frame duration of $2 \mathrm{~ms}$, the mobiles shall transmit their control information alternatively on each subcarrier so that the access node may refresh the channel state information once every 25 frames. The CEI scheduling algorithm relies on weights that set the dynamic priorities to allocate the resources. These weights are built in order to satisfy two major objectives: to maximise the system throughput and to encourage the nodes cooperation.

1) System Throughput Maximization Parameter: The CEI scheduler maximizes the system throughput in a MAC/PHY opportunistic approach. Data integrity requirements of the mobiles are enforced to adapt the modulation scheme and the transmission power to the mobile specific channel state. At each scheduling period, the scheduler computes the maximum number of bits $m_{k, n}$ that can be transmitted in a time slot of subcarrier $n$ if assigned to a mobile $k$, for all $k$ and all $n$. This number of bits is limited by two main factors: the data integrity requirement and the supported modulation orders.

The bit error probability is upper bounded by the symbol error probability and the time slot duration is assumed to be equal to the duration $T_{s}$ of an OFDM symbol [11]. The required received power $P_{r}(q, k)$ for transmitting $q$ bits in a RU while keeping below the data integrity requirement $B E R_{\text {target }, k}$ of the service flow of mobile $k$ is a function of the modulation type, its order and the single-sided power spectral density of noise $N_{0}$. For QAM and a modulation order $M$ on a flat fading channel [31]:

$$
P_{r}(q, k)=\frac{2 N_{0}}{3 T_{s}}\left[\operatorname{erfc}^{-1}\left(\frac{B E R_{\text {target }, k}}{2}\right)\right]^{2}(M-1),
$$

where $M=2^{q}$ and $\operatorname{erfc}$ is the complementary error function. $P_{r}(q, k)$ may also be determined in practice based on BER 
history and updated according to information collected on experienced BER.

The transmission power $P_{k, n}$ of mobile $k$ on subcarrier $n$ is upper bounded to a value $P_{\max }$ which complies with the transmission Power Spectral Density regulation:

$$
P_{k, n} \leq P_{\max }
$$

Given the channel gain $a_{k, n}$ experienced by mobile $k$ on subcarrier $n$ (including path loss and Rayleigh fading):

$$
P_{r}(q, k) \leq a_{k, n} P_{\max } .
$$

Hence, the maximum number of bits $q_{k, n}$ of mobile $k$ which can be transmitted on a time slot of subcarrier $n$ while keeping below its BER target is:

$$
q_{k, n} \leq\left\lfloor\log _{2}\left(1+\frac{3 P_{\max } \times T_{s} \times a_{k, n}}{2 N_{0}\left[\operatorname{erfc}^{-1}\left(\frac{B E R_{\text {target }, k}}{2}\right)\right]^{2}}\right)\right\rfloor .
$$

We further assume that the supported QAM modulation orders are limited so that $q$ belongs to the set $S=$ $\left\{0,2,4, \ldots, q_{\max }\right\}$. Hence, the maximum number of bits $m_{k, n}$ that will be transmitted on a time slot of subcarrier $n$ if this $\mathrm{RU}$ is allocated to the mobile $k$ is:

$$
m_{k, n}=\max \left\{q \in S, q \leq q_{k, n}\right\} .
$$

Opportunistic schedulers like MaxSNR based schemes allocate the resources to the mobiles which have the greatest $m_{k, n}$ values. This bandwidth allocation strategy maximizes the bandwidth usage efficiency but do not encourage the nodes cooperation. In order to extend the coverage area while preserving the system throughput maximization, a new parameter is added on $m_{k, n}$ which modulates this pure opportunistic resource allocation.

2) Incentive Parameter: The second major objective of the CEI is to incite nodes to participate to frame relay in order to extend the network coverage zone. This is achieved by extending the above cross-layer design to other layers. A new "Incentive Parameter" $\left(I P_{k}\right)$ is introduced based on the current estimation of the cooperation ratio:

$$
I P_{k}=\frac{R_{k}}{D_{k}}=\frac{D_{k}+\sum_{i=0 \ldots i=K}^{i} D_{k i}}{D_{k}},
$$

where $R_{k}$ is the global amount of data transmitted by mobile $k$. It is the sum between $D_{k}$, the amount of data transmitted to mobile $k$ for its own requirement and $D_{k i}$, the amount of data transmitted to the mobile $k$ for a mobile $i$ (then these data will be relayed to mobile $i$ by mobile $k$ in the relaying subframe). This information could be directly monitored by the access point, or signalled by each mobile to the access point.

We also define the cooperation ratio $C_{k}$ as the number of packets that mobile $k$ is ready to relay for other mobiles when it receives 100 packets for its own consumption, for example:

- when mobile $k$ relays no traffic out of the cell, $C_{k}$ equals $0 \%$;
- when it is ready to relay 50 packets out of the cell since it receives 100 packets for its own consumption, $C_{k}$ equals $50 \%$

- when the mobile relays as many packets out of the cell as its own received for its own consumption, $C_{k}$ equals $100 \%$.

Assuming that there are always packets to relay out of the cell, $I P_{k}$ will be respectively for these three cases equal to 1 , 1.5 and 2. Consequently, the resource allocation on $I P_{k}$ allows to give higher priority to mobiles that cooperate to extend the coverage zone with frame relaying.

3) Confidence Parameter: We assume that each mobile signals its $R_{k}$ and $D_{k}$ to the access point. Thanks to this information, the CEI scheduler will make adequate resource allocation rewarding the mobile according to its cooperation degree. However in order to block malicious mobiles that could lie on this information, we introduced a last parameter called the confidence parameter. The confidence parameter $T_{k}$ depends on the correspondence between the announced cooperative ratio and the observed forwarding ratio. This control is carried out by a monitor node (in our case the AP or cluster-head $(\mathrm{CH})$ ) in order to efficiently evaluate $T_{k}$. Unlike the existing monitoring mechanisms[19][20][16], the proposed solution is centralized and consequently is not impacted by the false evaluation related to the collision at the monitor node. Each $T_{k}$ varies between 0 and 1 included. When the access point monitoring $R_{k}$ and $D_{k}$ corresponds to the announced cooperative ratio, $T_{k}$ is set to 1 . Otherwise, when the mobile does not relay the announced amount of data for which it had previously received more priority, its $T_{k}$ is set to 0 for one round of scheduling in order to punish it. This ensures a deterrent threat for mobiles that would try to mislead the system.

4) Global CEI Algorithm Description: In the allocation process of a given time slot, the priority of a mobile $k$ for UR $n$ is determined by the magnitude of its CEI parameter:

$$
C E I_{k, n}=m_{k, n} \times \frac{R_{k}}{D_{k}} \times T_{k} .
$$

Based on the $m_{k, n}$ and $I P_{k}$ factor, the $C E I_{k, n}$ directly takes into account the channel states and the mobile behavior. Like MaxSNR, the physical layer information is used with $m_{k, n}$ in order to take advantage of the time, frequency and multiuser diversity and maximize the system capacity. However, contrary to existing schedulers, cooperation information as cooperation ratio $C_{k}$ is exploited in a weighted system with $I P_{k}$ parameter that introduces dynamic priorities between mobiles in order to ensure good rewards to mobiles that help extend the coverage zone. This results in an efficient scheme which guarantees a better network connectivity while avoiding tradeoff with the system capacity.

The $T_{k}$ parameter is an additional factor that allows to temperate $C E I_{k, n}$ value function of network confidence. Include $T_{k}$ parameter allows to be resistant to malicious nodes that would lie on their $\sum_{i=0 \ldots i=K}^{i} D_{k i}$. Thanks to this control 


\section{RU allocation of mobile 1 $C E I_{k, n}=m_{k, n} \times \mathbf{f}\left(C_{k}\right)$}

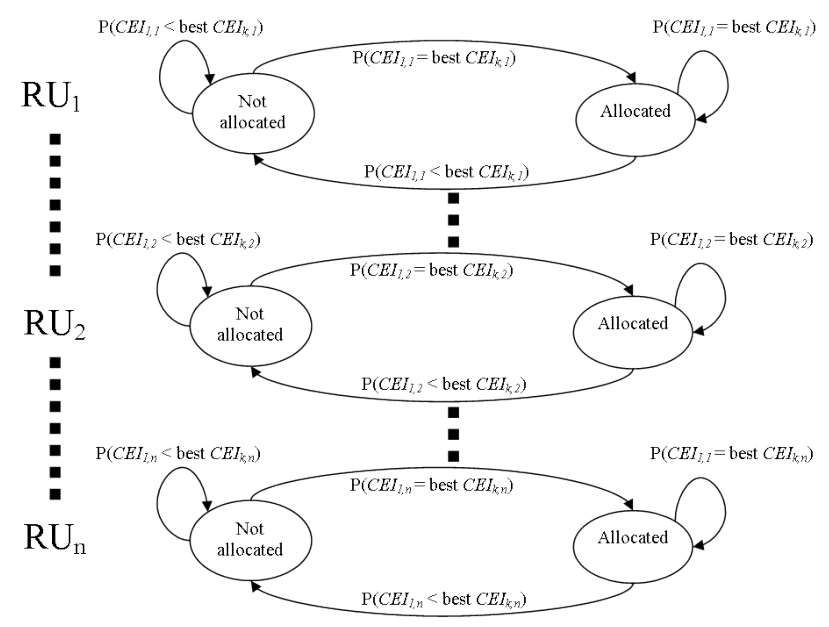

Fig. 4. Allocation probabilities for mobile 1 with CEI scheduler.

parameter, no mobile malicious behavior may provide benefits in terms of network resources.

As shown in Fig. 4, the probability for a mobile to receive Resource Units depends on the magnitude of its $C E I_{k, n}$ and consequently highly depends on the quantity of data relayed by the mobile to other mobiles in order to contribute to the coverage extension. The higher the cooperation ratio, the higher $I P_{k}$ and, unlike other schedulers, the higher the probability to receive bandwidth resources and to benefit from a low delay and a high throughput is. Consequently, with CEI algorithm, mobiles are encouraged to cooperate. If they want high priority and high QoS, they must not be selfish.

The CEI scheduling algorithm is detailed in Fig. 5. The scheduling is performed subcarrier by subcarrier and on a time slot basis for an improved granularity. In the allocation process of a given time slot, the priority of a mobile is determined by the magnitude of its CEI parameter. In the following items, we describe the proposed scheduling algorithm step by step.

- Step 0: The scheduler refreshes the current $m_{k, n}$ and updates cooperation ratio $I P_{k}$, confidence ratio $T_{k}$ and buffer occupancy $B O_{k}$ values. Then, it computes the $C E I_{k, n}$ parameter for each mobile and each subcarrier. Then, $n$ and $t$ are initialized to 1 .

- Step 1: For subcarrier $n$, the scheduler selects the mobile $k$ that has the greatest $C E I_{k, n}$ value. If $C E I_{k, n}$ is the same for several mobiles, the scheduler chooses the mobile that has the highest $B O_{k}$ value.

- Sub-step 1-1: If the virtual buffer occupancy ${ }^{1}$ of mobile $k$ is positive, the scheduler goes to Substep 1-2. Otherwise, if all virtual buffers are null or negative, the scheduler goes to Step 2. Otherwise, the scheduler selects the next mobile $k$ that has the greatest $C E I_{k, n}$ value and restarts Sub-step 11 (if $C E I_{k, n}$ is the same for several mobiles, the

\footnotetext{
${ }^{1}$ We define the virtual buffer occupancy as the current buffer occupancy of mobile $k$ minus the number of bits already allocated to this mobile.
}

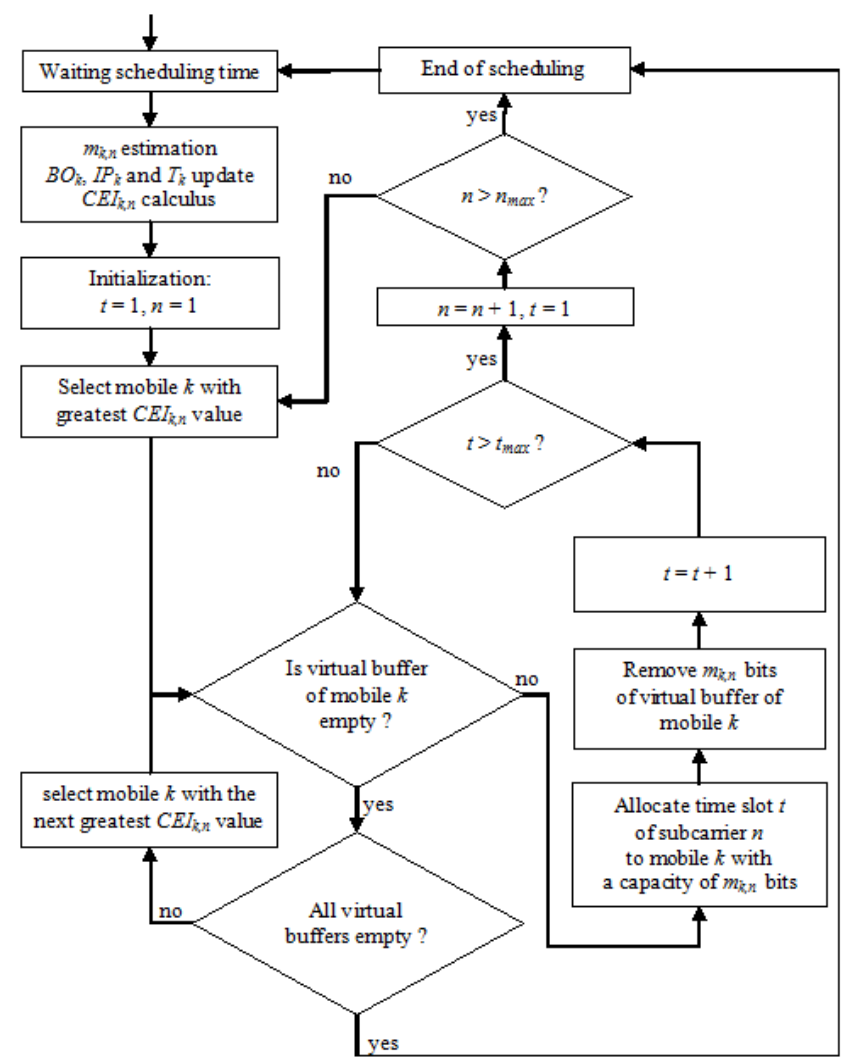

Fig. 5. CEI scheduling algorithm flow chart.

scheduler chooses the mobile that has the highest $B O_{k}$ value).

- Sub-step 1-2: The scheduler allocates time slot $t$ of subcarrier $n$ to mobile $k$ with a capacity of $m_{k, n}$ bits, removes $m_{k, n}$ bits of its virtual buffer and increments the value of $t$. If $t$ is smaller than the maximum number $t_{\max }$ of time slots by subcarrier, go to Sub-step 1-1 to allocate the following time slot. Otherwise, go to the following sub-step.

- Sub-step 1-3: Increment the value of $n$. If $n$ is smaller than the maximum number $n_{\max }$ of subcarriers, go to Step 1 to allocate the time slots of the next subcarrier. Otherwise, go to Step 2.

- Step 2: All buffers are empty or all time slots of all subcarriers are allocated and the scheduling ends.

5) Discussion: We propose to limit $I P_{k}$ values to a maximum of 2 that corresponds to a cooperation ratio of $100 \%$. Indeed, we assume that a mobile with a $C_{k}$ value higher than $100 \%$ could be considered irrational. Indeed, it could be a problem that a mobile relays more packets than it receives for its own consumption. We consider that it could be not profitable for it and also for the system since a mobile with a disproportionate cooperation ratio could quickly use its battery and obtain all resources in the cell and this will excessively penalize other mobiles, even those that have a good cooperation ratio. 


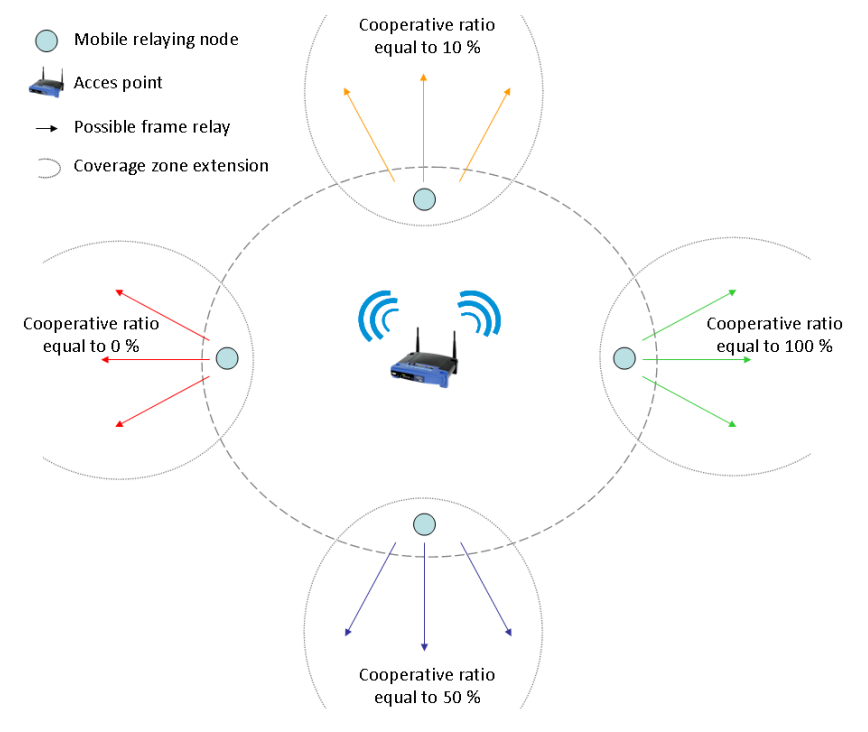

Fig. 6. Simulation setup.

\section{Performance Analysis}

In this section we evaluate the performance of the proposed CEI scheduling and we compare it to the classical Round Robin allocation and the well known MaxSNR scheduler. We consider four kinds of nodes: the selfish nodes that do not relay any packet $\left(C_{k}=0 \%\right)$, the nodes that relay a few packets with $C_{k}=10 \%$, the nodes that are more cooperative with $C_{k}=50 \%$ and the nodes that are really network friendly with a maximum cooperative ratio of $100 \%$. We focus on two main performance metrics: the mean packet delay and the mean throughput provided at each mobile. Performance evaluation results are obtained using OPNET discrete event simulations with the simulation parameters presented in the next subsection.

\section{A. Simulation setup}

The scenario of the simulation is illustrated in figure 6 .

We assume that each frame is formed by 128 subcarriers and 5 time slots. We select 128 subcarriers for each frame in order to make the proposed system compatible with IEEE $802.11 \mathrm{n}$ where the channel is divided into 128 subcarriers (for $40 \mathrm{MHz}$ transmission). The channel gain model on each subcarrier considers free space Path Loss $a_{k}$ and multipath Rayleigh fading $\alpha_{k, n}^{2}$ :

$$
a_{k, n}=a_{k} \times \alpha_{k, n}^{2} .
$$

where $a_{k}$ is dependent on the distance between the access point and mobile $k$ and $\alpha_{k, n}^{2}$ represents the flat fading experienced by mobile $k$ on subcarrier $n . \alpha_{k, n}$ is Rayleigh distributed with an expectancy equal to one [32]. Additionally, the maximum transmission power satisfies:

$$
10 \log _{10}\left(\frac{P_{\max } T_{s}}{N_{0}} \times a_{\text {ref }}\right)=31 \mathrm{~dB}
$$

and BER target is equal to $10^{-3}$. With this setting, the value of $m_{k, n}$ is 3 bits when $\alpha_{k, n}^{2}$ equals one.

We consider that all mobiles run the same videoconference application. This demanding type of application generates a high volume of data with a high sporadicity and requires tight delay constraints which substantially complicate the task of the scheduler. Each traffic is composed of an MPEG-4 video stream [33] and an AMR voice stream [34]. The traffic load variation is carried out by increasing the mobile bit rate requirement of each mobile all together.

\section{B. Delay impacts}

First we focus on the mean mobile packet delay provided by each scheduler according to different traffic loads, paying close attention to their ability to encourage the mobile cooperation with low delay guaranteed. The obtained results are plotted in figure Fig. 7 with the mean throughput required by each mobile of the cell represented on the abscissa.

Figure 7(a) shows the case of RR with different cooperation ratios of nodes. We remark that the classical RR fails to promote cooperation activities. The RR fairly allocates the RUs to the mobiles without taking into account the effort of the cooperative mobile nodes that share their allocated resources with other nodes located out of the primary access point cell. Consequently, the more cooperative the nodes are, the less resources for their own transmission they have. Moreover, the RR does not benefit from multiuser diversity which results in a bad utilization of the bandwidth and consequently, a poor system throughput. Thus, an unacceptable packet delay is experienced even with relatively low traffic loads.

Figure 7(b) illustrates the obtained results in the case of MaxSNR with different cooperation ratios of nodes. We point out that even if a higher traffic load is supported with an acceptable packet delay, the cooperative nodes are not rewarded and their performance in terms of QoS are inferior to those of the non-cooperative nodes.

Our recent proposed scheduling algorithm, WFO, Figure 7(c) gives the beginning of a solution. It guarantees the same QoS to each mobile whatever the context (all the curves are superimposed). Consequently, cooperative mobiles are not penalized in terms of mean packet delay. The only cost to pay to help network extension by relaying frame to other mobiles is energy consumption. However, the energy consumed by the cooperative nodes must not be ignored. That's why the proposed CEI rewards the cooperative mobile nodes according to their cooperation ratio. Figure 7(d) shows the obtained results in the case of the scheduler: CEI. We remark that CEI does not only encourage the nodes to cooperate but also enhances the performance in terms of delay. When the nodes increase their cooperation ratio, the enhancement of their delay is more important. For example, the nodes with $100 \%$ as cooperative ratio, have a delay inferior to $100 \mathrm{~ms}$ when the mean required throughput is less than $3 \times 10^{5}$ bps which is not possible with other schedulers. The CEI dynamically and gradually adjusts the relative priorities of the mobiles in order to fairly and adequately reward them according to their relative cooperation ratio. With this approach, sparingly delaying the selfish mobiles, the CEI helps the others and whatever the traffic load, the mobile that provides the best cooperative ratio experiences the lowest packet delay. This adequately compensates the supplementary energy consumption of a network friendly behavior. 


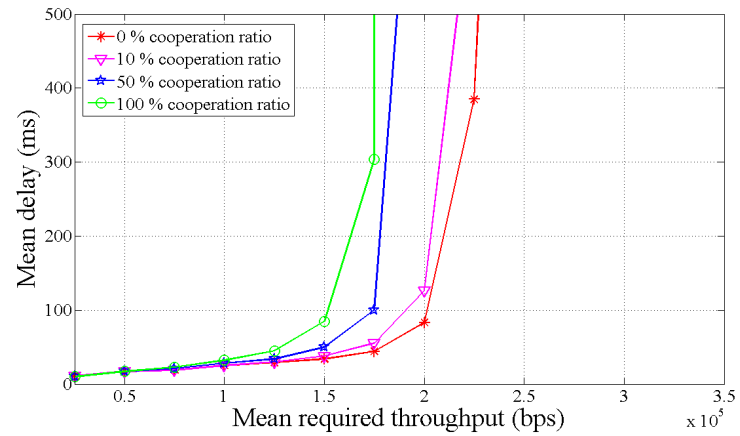

(a) With RR.

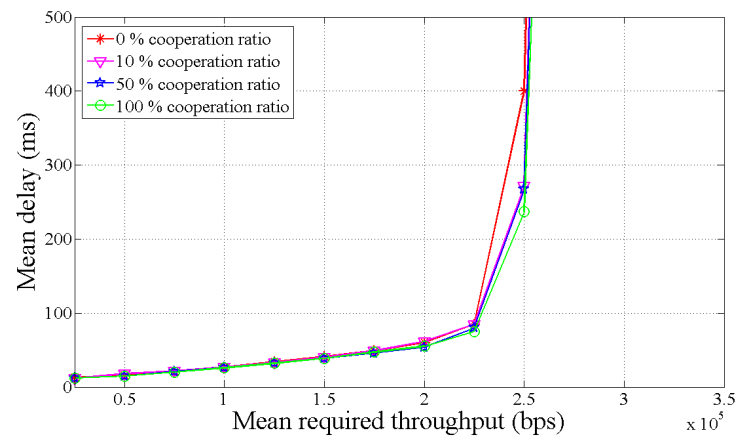

(c) With WFO.

Fig. 7. Measured mobile mean delay with respect to their cooperation ratio.

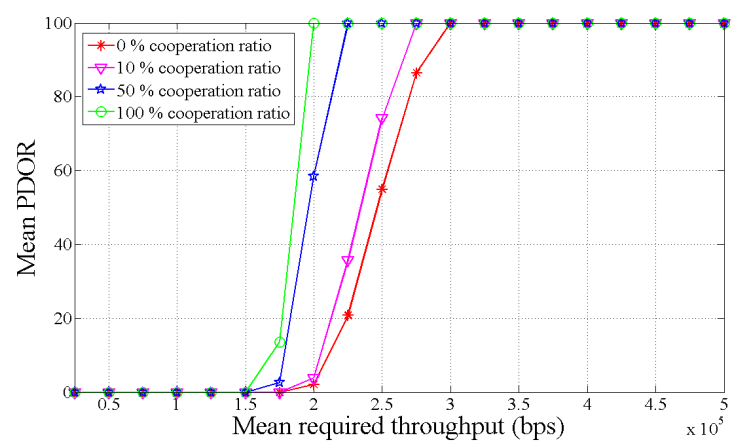

(a) With RR.

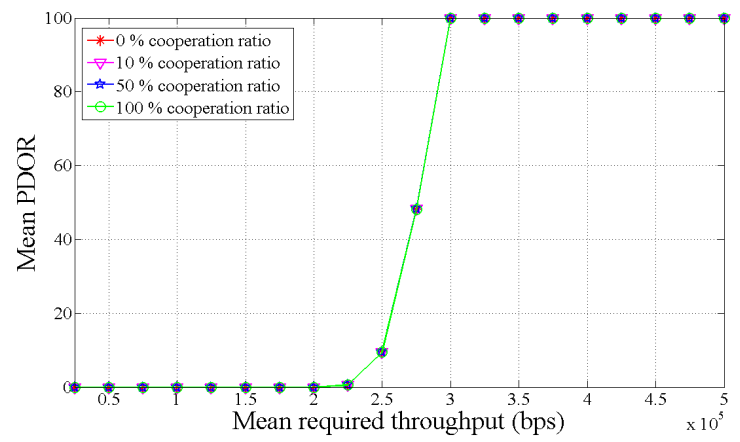

(c) With WFO.

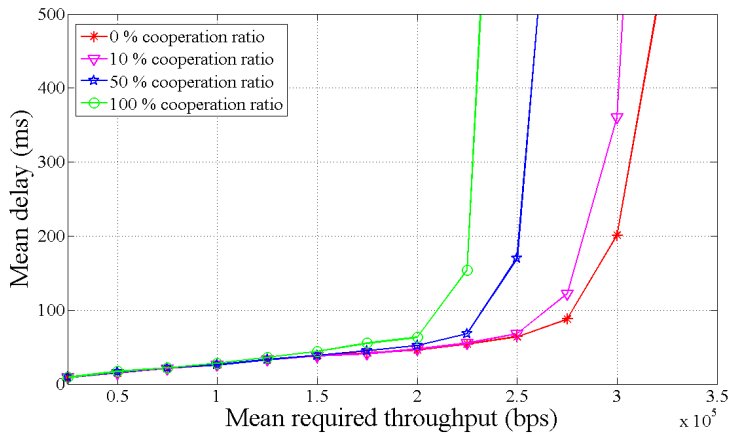

(b) With MaxSNR.

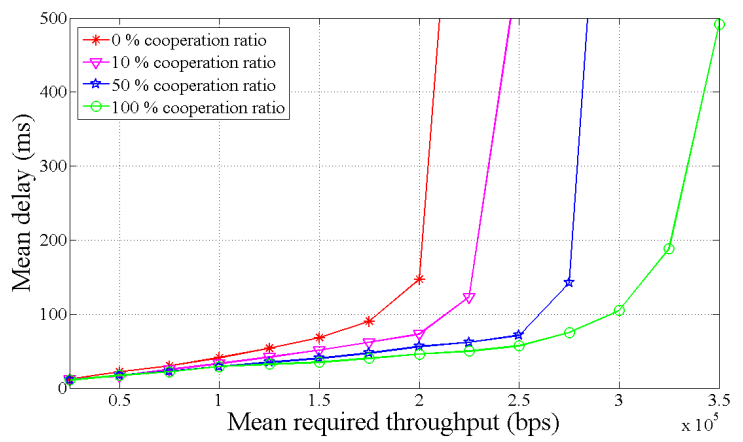

(d) With CEI.

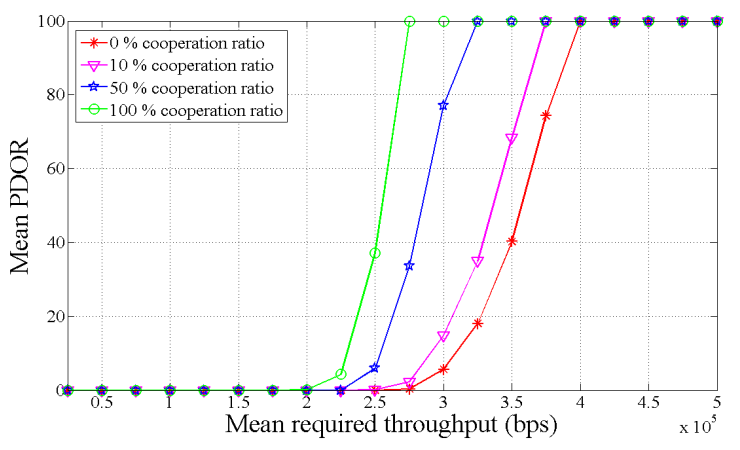

(b) With MaxSNR.

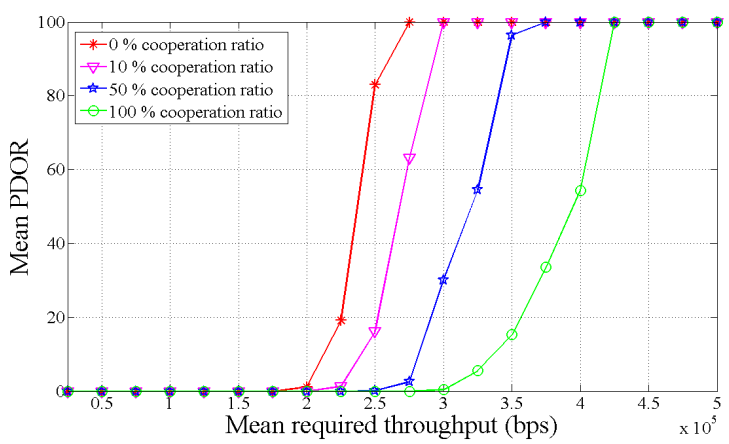

(d) With CEI.

Fig. 8. Measured mobile dissatisfaction with respect to their cooperation ratio. 


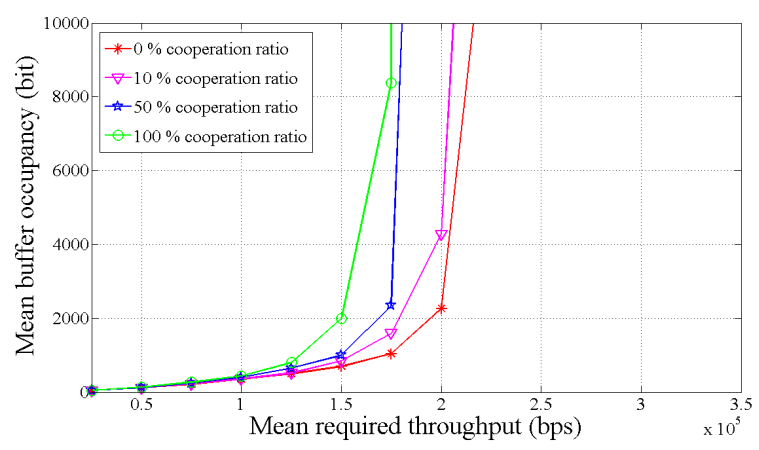

(a) With RR.

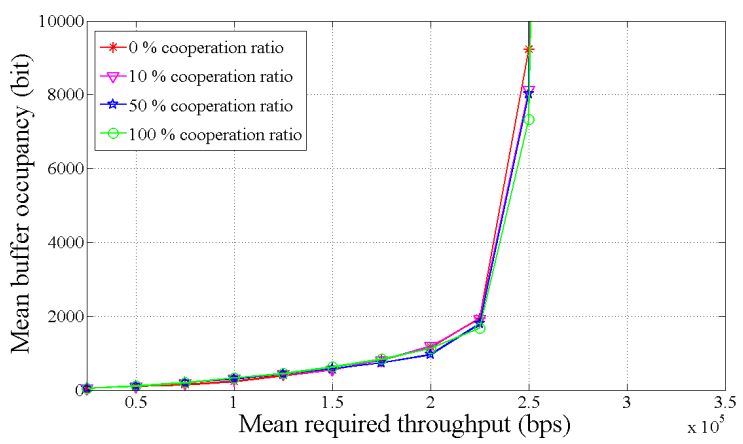

(c) With WFO.

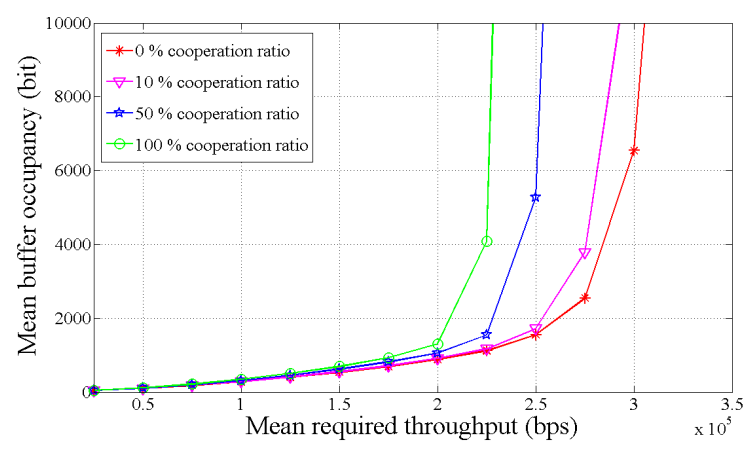

(b) With MaxSNR

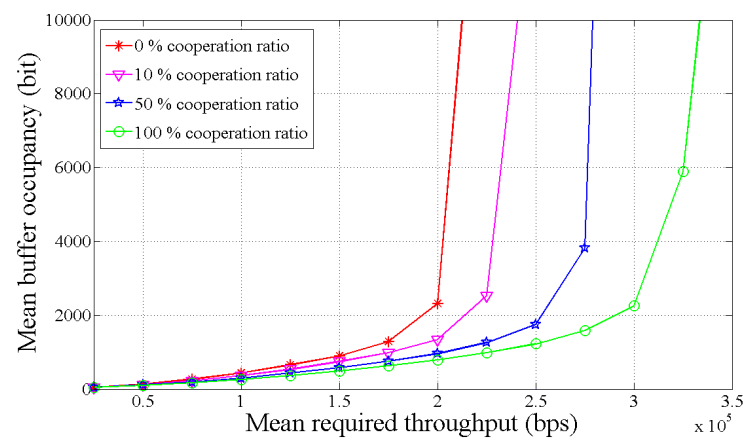

(d) With CEI.

Fig. 9. Measured moibile mean buffer occupancy with respect to their cooperation ratio.

\section{Buffer occupancy and PDOR impacts}

The obtained results regarding the mean packet delay outage ratio (fig. 8) and the mean buffer occupancy (fig. 9) corroborate with the analysis in the previous subsection.

As expected, classical RR yields bad results. Indeed, since multiuser diversity is not exploited, the overall spectral efficiency and system throughput are low. Consequently, the delay thresholds are widely exceeded and the mobiles are dissatisfied while the buffers are quickly filled. The mobile satisfaction is directly impacted and the PDOR gives high values even with a low traffic. More generally, the higher the cooperation ratio of a mobile is, the more it will face difficulties.

MaxSNR, WFO and CEI opportunistic schedulers take into account the wireless specificities, increasing system capacity and providing better results. However, MaxSNR is highly unfair and still gives inadequate priorities, such as RR. It fully satisfies the required QoS of selfish mobiles at the expense of the satisfaction of friendly mobiles that encourage the network extension.

In contrast, WFO reduces this severe lack of fairness and each mobile benefits from the same QoS which results in the same mean buffer occupancy and the same mean PDOR. In order to compensate the supplementary energy consumption generated by each relay, the CEI rewards the mobiles according to their behavior. The higher the cooperation ratio of a mobile is, the less it will face difficulties.

\section{Throughput impacts}

We will now have a look at the mean mobile throughput provided by each scheduler according to the different traffic loads paying a special attention to their ability to encourage mobile cooperation with a high guaranteed throughput. The obtained results are plotted in figure 10. The first parts of these four figures, where all the curves are superimposed, correspond to an unoverloaded system. Each mobile can be served and each scheduler is able to provide the required throughput.

In the second parts of these figures, the system capacity is exceeded and the scheduler has to make a choice. With RR the system capacity goes past its limit when each source requires 200 Kbps. With MaxSNR and CEI which provide an efficient spectral efficiency thanks to their opportunistic approaches, this limit is set to $250 \mathrm{Kbps}$. However, with WFO, this limit is higher because the multi-user diversity is better used.

In an overloaded context, clearly, RR and MaxSNR give advantage to the selfish mobile nodes as illustrated in figures 10(a) and 10(b). Indeed, with these schedulers, each mobile of the primary access point coverage receives the same mean number of RUs. However, a mobile with a cooperation ratio of $100 \%$ only keeps the half of its allocated RUs for its own consumption while a selfish mobile with a $C_{k}$ of $0 \%$ keeps all its allocated RUs for its own requirements. Consequently, the friendly mobile with $C_{k}$ equal to $100 \%$ has a personal provided throughput half lower than the one of the selfish mobile. This result is a really disheartening situation for cooperative mobiles that are eventually penalized ${ }^{2}$.

Again in an overloaded context, WFO (Fig 10(c)), provides

${ }^{2}$ The penalty is proportional to the $C_{k}$ magnitude. For example, when $C_{k}$ equals $50 \%$, the mobile forwards 50 packets when it receives 100 packets for its own consumption. Consequently, its personal provided throughput is a third lower than the one of the selfish mobile. 


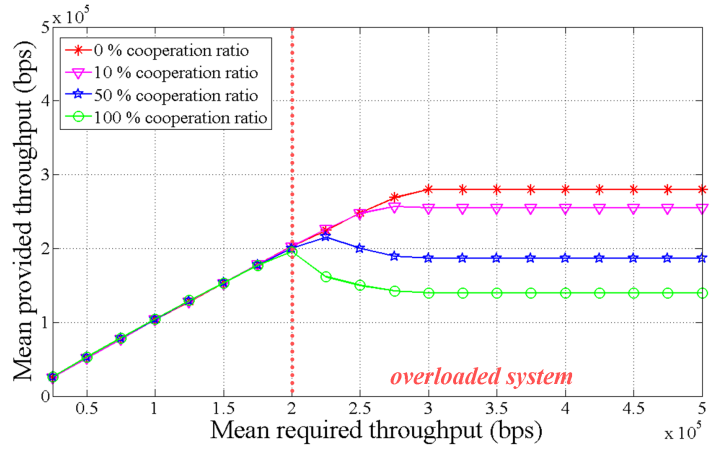

(a) With RR.

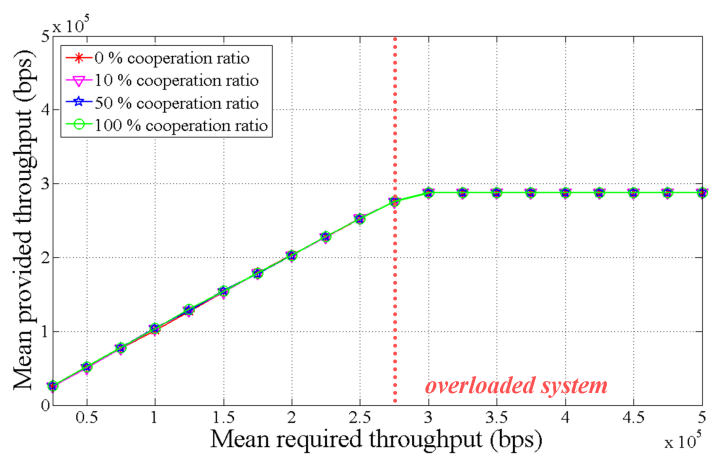

(c) With WFO.

Fig. 10. Measured mobile throughput with respect to their cooperation ratio.

a fair management between mobiles whatever their cooperation ratio. This results in the same provided throughput for all mobiles whatever the traffic load. At last, friendly mobiles are not penalized even if they are not rewarded for their good behaviour either. Unlike these schedulers, the CEI does not deploy the same strategy while reaching the overloaded limit with the same traffic load as MaxSNR as illustrated in figure 10(d). The more network friendly a mobile is and relays packets in order to help primary access point coverage extension, the more the CEI increases its priority. Consequently, when the CEI can not serve all mobiles, it first sacrifices the selfish mobiles, then the next least friendly mobiles. The result of this new scheduling strategy is that mobiles are encouraged to cooperate to keep a high throughput.

\section{E. Relay efficiency impacts}

Figure 11 illustrates the relay efficiency in terms of the total mean throughput that each scheduling algorithm has allowed to provide out of the cell ${ }^{3}$. We remark that RR provides the worst performances compared to MaxSNR, WFO and CEI. MaxSNR allows to relay more packets but it is the CEI which gives the best number of provided throughputs out of the cell. In addition, we can observe that the mean provided throughput offered by MaxSNR and RR decrease when the system capacity is reached. This is due to an unfair and high penalty of the best cooperative mobile. WFO gives better performance results than RR and MaxSNR. CEI gives

\footnotetext{
${ }^{3}$ The cell can be assimilated to the primary access point coverage zone without assuming relaying.
}

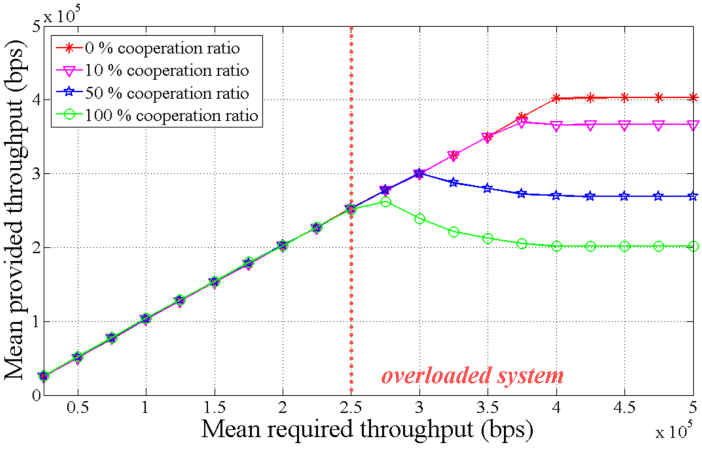

(b) With MaxSNR.

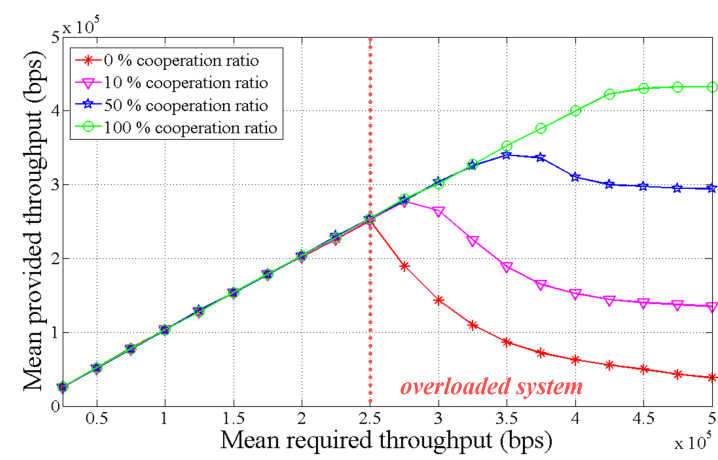

(d) With CEI.

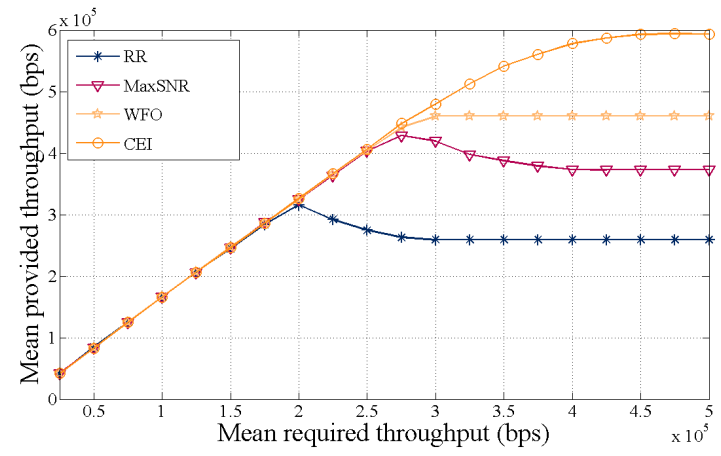

Fig. 11. Relay efficiency.

anyway the best performance results. The CEI, according more priority to friendly mobiles, continues to increase the total amount of forwarding throughputs until a high traffic load which corresponds to a high network extension capacity. With this new resource allocation strategy, when the mean required throughput of each mobile is equal to $500 \mathrm{Kbps}$, the total amount of data transmitted out of the cell in order to extend the coverage area can be increased around 59\% compared to the well acknowledged MaxSNR and around 129\% compared to the classical scheduling algorithm RR.

\section{F. Results summarization}

Figure 12 concludes these performance evaluations. We notice that for a high traffic load of $500 \mathrm{Kbps}$ for each mobile, the scheduler behaviour showing the mean cell mobile 


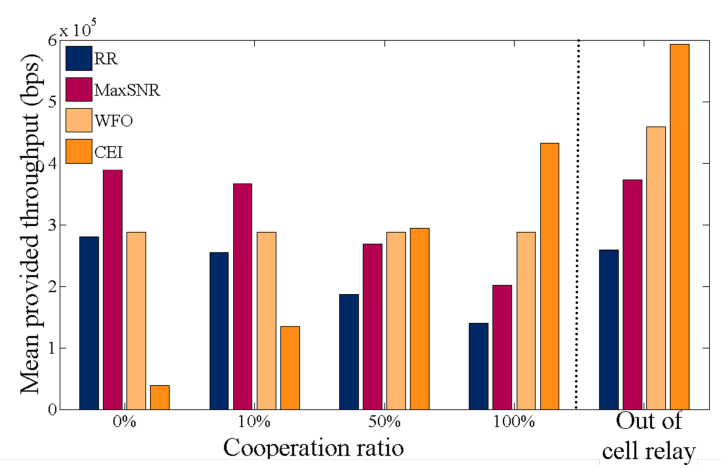

Fig. 12. Provided throughput function of cooperation ratio in overload system.

provided throughput according to their cooperation ratio and the total mean provided throughput out of the cell (on the right). These latter results clearly corroborate the previous results.

With RR and MaxSNR schedulings, there is no interest for a mobile to cooperate. To be friendly induces to increase its mean packet delay as illustrated in figures 7 (a) and 7(b), but also to reduce its potential throughput particularly in an overloaded context (Fig. 10(a) and 10(b)). Unlike RR and MaxSNR, there is a significant interest for a mobile to cooperate with CEI. To be friendly induces to decrease its mean packet delay whatever the traffic load on the system (Fig. 7(d)) but also allows to increase its potential throughput in an overloaded context (Fig. 10(d)). Thanks to this new resource allocation strategy, mobiles are not penalized anymore when they cooperate but receive high rewards in terms of QoS which could easily compensate their cooperative energy cost. For a high traffic load of $500 \mathrm{Kbps}$ for each mobile, the cooperative mobiles can increase their own throughput by around $114 \%$ compared to MaxSNR and by around 209\% compared to RR resource allocation strategy. Therefore, this allows a significant coverage extension which was not achieved with RR and MaxSNR strategies and free mobiles.

\section{CONClusion}

In this paper we proposed a new protocol based on an incentive approach and a scheduling algorithm in order to reward cooperative nodes and extend the wireless area coverage. This incentive approach encourages nodes to relay neighbours' frames by increasing their priority to access resources' allocation. In addition, the cross-layer approach is used in order to optimize the QoS parameters. With our proposed scheme, a mobile remains free to cooperate or not but the proposed CEI scheduler sparingly rewards participating nodes so that it is more attractive for them to actively contribute to a high network coverage. This results is a well-balanced resource allocation which allows an increase in the network coverage area while never reduces the global system throughput. These optimistic results are attributed to a combined opportunistic approaches that help the system reaches a balanced state. A minimum throughput is guaranteed to all mobiles of the cell and, thanks to its high spectral efficiency, the mean packet delay provided to the selfish mobiles by having the CEI staying close to the best RR performance. Moreover, the simulation results show that the proposed solution gives better results than the available scheduling algorithms like MaxSNR and WFO. These CEI interesting performance results show that a significant priority is given to mobiles which help the network provides a low packet delay and a high personal throughput. In future work, we plan to introduce services' differentiation in our proposed solution.

\section{REFERENCES}

[1] A. K. Sadek, Z. Han, and K. Ray Liu, "Distributed relay-assignment protocols for coverage expansion in cooperative wireless networks," IEEE Transactions on Mobile Computing, vol. 9, no. 4, pp. 505 - 515, 2010.

[2] A. Florea and H. Yanikomeroglu, "The optimal number of hops in infrastructure-based fixed relay networks," in Proc. IEEE Globecom, St. Louis, MO, Nov. 2005, pp. 3242- 3247.

[3] M. Sikora, J. N. Laneman, M. Haenggi, D. Costello, and T. Fuja, "Bandwidth and power-efficient routing in linear wireless networks," IEEE Transactions Inf. Theory, vol. 52, no. 6, pp. 624- 2633, 2006.

[4] L. Xiao, T. E. Fuja, and D. J. Costello, "Mobile relaying: Coverage extension and throughput enhancement," IEEE Transactions on Communications, vol. 58, no. 9, pp. 2709 - 2717, 2010.

[5] B. Bakaimis and T. Lestable, "Connectivity investigation of mobile relays for the next generation wireless systems," in Proc. of 61th IEEE Vehicle Technology Conference (VTC-Springer), Stockholm, Sweden, Nov. 2005, pp. 2192- 2195.

[6] L. Chen, L. Libman, and J. Leneutre, "Conflicts and incentives in wireless cooperative relaying: A distributed market pricing framework," In IEEE Transactions on Parallel and Distributed Systems, vol. 22, no. 5, pp. $758-772,2011$.

[7] L. Depeng, X. Youyun, and L. Jing, "Distributed relay selection over multi-source and multi-relay wireless cooperative networks with selfish nodes," Computer Communications, vol. 33, no. 17, pp. 2145-2153, 2010.

[8] L. Dapeng, X. Youyun, and M. Xinbing, W. Guizani, "Coalitional game theoretic approach for secondary spectrum access in cooperative cognitive radio networks," IEEE Transactions on Wireless Communications, vol. 10, no. 3, pp. 844-856, 2011.

[9] L. Dapeng, L. Youyun, X. Jing, W. Xinbing, and M. Guizani, "A spatial game for access points placement in cognitive radio networks with multi-type service," in Proceedings of IEEE Global Telecommunications Conference, 2010.

[10] J. Nagle, "On packet switches with infinite storage," IEEE Transactions on Communications, vol. 35, no. 4, pp. 435 - 438, April 1987.

[11] C. Y. Wong and R. S. Cheng, "Multiuser OFDM with adaptive subcarrier, bit, and power allocation," IEEE J. Sel. Areas Commun., 1999.

[12] X. Wang and W. Xiang, "An OFDM-TDMA/SA MAC protocol with QoS constraints for broadband wireless LANs," ACM/Springer Wireless Networks, vol. 12, no. 2, pp. 159 - 170, 2006.

[13] C. Gueguen and S. Baey, "A fair opportunistic access scheme for multiuser OFDM wireless networks," Journal on Wireless Communications and Networking. European Association for Signal Processing (EURASIP). Special issue: Fairness in Radio Resource Management for Wireless Network, Feb. 2009.

[14] A. Nosratinia, T. Hunter, and A. Hedayat, "Cooperative coomunication in wireless networks," IEEE Communications Letters, vol. 42, no. 10, pp. $74-80,2004$.

[15] P. Michiardi and R. Molva, "Core: a collaborative reputation mechanism to enforce node cooperation in mobile ad hoc networks," in Proceedings of the IFIP TC6/TC11 Sixth Joint Working Conference on Communications and Multimedia Security: Advanced Communications and Multimedia Security, 2002, pp. 107- 121.

[16] S. Marti, T. J. Giuli, K. Lai, and M. Baker, "Mitigating routing misebehaviour in mobile ad-hoc networks," in Proceedings of the 6th annual international conference on Mobile computing and networking (MobiCom'00), 2000.

[17] S. Buchegger and J. Le Boudec, "Performance analysis of the cofidant protocol," in Proceedings of 3rd ACM international Symposium on Mobile ad hoc networking \& computing, 2002, pp. 226- 236.

[18] S. Bansal and M. Baker, "Observation-based cooperation enforcement in ad hoc networks," in CoRR, 2003. 
[19] A. Rachedi and A. Benslimane, "Toward a cross-layer monitoring process for mobile ad hoc networks," Security and Communication Networks, vol. 2, no. 4, pp. 351-368, 2009.

[20] — "Cross-layer approach to improve the monitoring process for mobile ad hoc networks based on ieee 802.11," in Proceedings of 50th IEEE Global Telecommunications Conference (GLOBECOM'07), 2007.

[21] L. Yun, W. Xinbing, and M. Guizani, "Resource pricing with primary service guarantees in cognitive radio networks: A stackelberg game approach," in Proceedings of IEEE Global Telecommunications Conference, 2009

[22] Y. Xiao, G. Bi, and D. Niyato, "Game theoretic analysis for spectrum sharing with multi-hop relaying," IEEE Transactions on Wireless Communications, vol. 99, pp. 1-11, 2011.

[23] L. Buttyan and J. P. Hubeaux, "Enforcing service availability in mobile ad-hoc wan," in Proceedings of the 1st ACM international symposium on Mobile ad hoc networking \& computing, 2000, pp. 87-96.

[24] Z. Li and H. Shen, "Analysis of a hybrid reputation management system for mobile ad-hoc networks," in Proceedings of International Conference on Computer Communications and Networks, 2009.

[25] G. Mergen and L. Tong, "Random scheduling medium access for wireless ad hoc networks," in Proc. IEEE Int. Conf. on MILCOM, vol. 2 , October 2002, pp. $868-872$

[26] C. Gueguen and S. Baey, "An efficient and fair scheduling scheme for multiuser OFDM wireless networks," in Proc. IEEE Int. Wireless Communications and Networking Conference (WCNC), March. 2008, pp. $1610-1615$.

[27] M. Andrews, K. Kumaran, and K. Ramanan, "Providing quality of service over a shared wireless link," IEEE Communications Magazine, vol. 39, pp. 150-154, Feb. 2001.

[28] V. de Beek et al., "A time and frequency synchronization scheme for multiuser OFDM," IEEE J. Sel. Areas Commun., vol. 17, pp. 1900 1914, Nov. 1999.

[29] Y. G. Li, N. Seshadri, and S. Ariyavisitakul, "Channel estimation for OFDM systems with transmitter diversity in mobile wireless channels," IEEE J. Sel. Areas Commun., vol. 17, no. 3, pp. 461 - 471, March. 1999.

[30] T. E. Truman and R. W.Brodersen, "A measurement-based characterization of the time variation of an indoor wireless channel," in Proc. IEEE Int. Universal Personal Communications Record (ICUPC), vol. 1, Oct. 1997, pp. $25-32$.

[31] J. G. Proakis, Digital Communications. 3rd ed. New York: McGrawHill, 1995.

[32] J. D. Parsons, The Mobile Radio Propagation Channel. Wiley, 1992.

[33] S. Baey, "Modeling MPEG4 video traffic based on a customization of the DBMAP," in Proc. Int. Symposium on Performance Evaluation of Computer and Telecommunication Systems (SPECTS), July 2004.

[34] P. Brady, "A model for generating on-off speech patterns in two-way conversation,” Bell System Technical Journal, vol. 48, 1969.

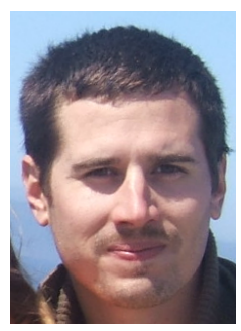

Cédric Gueguen received his research MS degrees in computer science from the University of Paris VI (UPMC) in France in 2006. He received his $\mathrm{Ph} . \mathrm{D}$. degree in computer science from the UPMC in 2010 while working in the Networks and Performance Analysis (NPA) group at the Laboratory of Paris 6 (LIP6). In 2010, he was assistant professor (ATER) at the university Paris-Est Marne-la-Vallée (UPEMLV) in "Protocols Architecture \& Software for Networks team" (PASNET). He was a member of the Gaspard Monge Computer Science laboratory

(LIGM) during 2010-2011.

$\mathrm{He}$ is currently working as associate professor (matre de confrences) at the university of Rennes 1 and he is a member of the IRISA laboratory since September 2011.

His research interests lie in the field of wireless networking, scheduling algorithms, fairness issues, Quality of Service (QoS), Quality of Experience (QoE). He participated or still participates to the WIP international research project (2006-2009).

Dr. Gueguen serves on the technical program committee of several IEEE conferences, including Globecom, ICC, WiMob, WCNC, PIMRC, VTC...

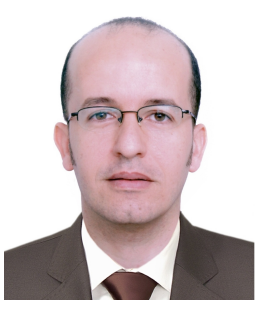

Abderrezak Rachedi received his engineer degree in computer science from the university of technology and science Houari Boumedienne (USTHB), Algiers, Algeria, in 2002 and research MS and professional MS degrees in computer science from the University of Lyon and University of Savoie in France in 2003 and 2005, respectively. He received his Ph.D. degree in computer science from the university of Avignon, France, in 2008.

He was assistant professor (ATER) at the university Paris-Est Marne-la-Vallée (UPEMLV) in 2008. He is currently working as associate professor (maître de confèrences) at the university Paris-Est Marnela-Vallée (UPEMLV) and a member of the Gaspard Monge Computer Science laboratory (LIGM CNRS UMR 8049) since September 2009.

His research interests lie in the field of wireless networking, wireless sensor networks, security protocols, intrusion detection systems, network monitoring mechanisms, anonymity protocols, fairness protocols, distributed algorithms, quality of services with security, inter-vehicular communications. He participated or still participates to several national and international research projects. Among them ANR CLADIS (2006-2009), Super-BQR MWSN (2009-2010), Digiteo ViSuNet (2010-2013), PPS-WSNTM (2011-2014).

Dr Rachedi serves as Associate Editor of Wireless Communications and Mobile Computing (WCMC) journal and he also served on the Editorial Boards of Journal of Computer Systems, Networks, and Communications (JCSNC). He has been on the technical program committee of different ACM and IEEE conferences, including Globecom, ICC, WiMob, WCNC, IWCMC and chaired some of their sessions. Dr Rachedi is an IEEE member.

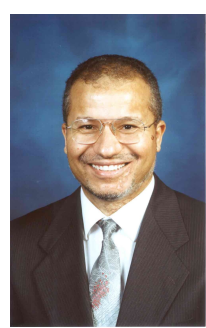

Mohsen Guizani (S'85-M'89-SM'99-F'09) is currently a Professor and the Associate Vice President for Graduate Studies at Qatar University, Doha, Qatar. He was the Chair of the Computer Science Department at Western Michigan University from 2002 to 2006 and Chair of the Computer Science Department at University of West Florida from 1999 to 2002. He also served in academic positions at the University of Missouri-Kansas City, University of Colorado-Boulder, Syracuse University and Kuwait University. He received his B.S. (with distinction) and M.S. degrees in Electrical Engineering; M.S. and Ph.D. degrees in Computer Engineering in 1984, 1986, 1987, and 1990, respectively, from Syracuse University, Syracuse, New York.

His research interests include Computer Networks, Wireless Communications and Mobile Computing, and Optical Networking. He currently serves on the editorial boards of six technical Journals and the Founder and EIC of "Wireless Communications and Mobile Computing" Journal published by John Wiley (http://www.interscience.wiley.com/jpages/1530-8669/). He is the author of eight books and more than 300 publications in refereed journals and conferences. He guest edited a number of special issues in IEEE Journals and Magazines. He also served as member, Chair, and General Chair of a number of conferences.

Dr. Guizani served as the Chair of IEEE Communications Society Wireless Technical Committee (WTC) and Chair of TAOS Technical Committee. He was an IEEE Computer Society Distinguished Lecturer from 2003 to 2005. Dr. Guizani is an IEEE Fellow and a Senior member of ACM. 